www.nature.com/jhg

\title{
EDITORIAL
}

\section{Epigenomics comes of age with expanding roles in biological understanding and clinical application}

\author{
Journal of Human Genetics (2013) 58, 395; doi:10.1038/jhg.2013.70
}

$\mathrm{E}$ pigenetics is a mechanism that regulates gene expression not depending on the underlying DNA sequence, but on the chemical modification of DNA or histone proteins. Thanks to current advanced technologies, the understanding of epigenetic modifications or 'epigenome' in the human genome has been growing rapidly.

Epigenetics initially contributed to the understanding of congenital diseases, because of the hallmark epigenetic phenomena such as genomic imprinting and X-chromosome inactivation, which had been identified in mice, fitted well to a subset of human congenital diseases such as Prader-Willi, Angelman and Beckwith-Wiedemann syndromes. These findings were utilized soon after in clinics as epigenetic testing. ${ }^{1}$

Newly identified molecules related to DNA methylation have meant the contribution of epigenetics has expanded to the understanding of various congenital diseases. ${ }^{2}$ Furthermore, a combination of the recent studies into molecules related to histone modification and advances in next-generation technology has led to the conclusion that many of the congenital diseases in which genetic cause has not been identified are caused by mutations of the genes that encode histonemodification enzymes, such as in Kleefstra syndrome. ${ }^{3}$

Epigenetic studies are now extensively conducted in cancer science, and epigenetic approaches are utilized to further understand complex or multifactorial diseases, including diabetes mellitus, obesity and mental disease, because the epigenetic mechanism is susceptible to environmental factors such as nutrition and mental stress. ${ }^{4,5}$ Through various animal studies, these acquired epigenetic alterations are now believed to be inherited by the next generation. This concept is a challenge to the theory of Darwinism that has been believed by biologists and genetic researchers for over hundred years. ${ }^{6}$

Under these circumstances, we are pleased to have this opportunity to compile a special section for the July issue of the Journal of Human Genetics. We guest editors would like to thank Professor Tokunaga, editor of this journal, for this great opportunity. We are very happy and proud of the papers contributed for this section, including the eight review articles by top-level epigenetic researchers in the United States and Japan. Covering various topics of epigenetic research, both basic and clinical, these reviews should provide readers with a comprehensive overview and understanding of the status of current epigenetic studies. The two original articles also make a very valuable contribution to this section.

We hope this collection of papers will help readers gain a better understanding of what has been done and what is yet to be done in the field of epigenetics, and how this subject is intimately associated with our everyday life.

Takeo Kubota ${ }^{1}$ and Kenichiro Hata ${ }^{2}$ ${ }^{1}$ Department of Epigenetic Medicine, Faculty of Medicine, University of Yamanashi, Chuo, Japan and ${ }^{2}$ Department of Maternal-Fetal Biology, National Research Institute for Child Health and Development, Tokyo, Japan E-mail: takeot@yamanashi.ac.jp

1 Kubota, T., Das, S., Christian, S. L., Baylin, S. B., Herman, J. G. \& Ledbetter, D. H. Methylation-specific PCR simplifies imprinting analysis. Nat. Genet. 16, 16-17 (1997).

2 Hata, K., Okano, M., Lei, H. \& Li, E. Dnmt3L cooperates with the Dnmt3 family of de novo DNA methyltransferases to establish maternal imprints in mice. Development 129, 1983-1993 (2002).

3 Kleefstra, T., Kramer, J. M., Neveling, K., Willemsen, M. H. Koemans, T. S. Vissers, L. E. et al. Disruption of an EHMT1-associated chromatin-modification module causes intellectual disability. Am. J. Hum. Genet. 91, 73-82 (2012).

4 Kucharski, R., Maleszka, J., Foret, S. \& Maleszka, R. Nutritional control of reproductive status in honeybees via DNA methylation. Science 319, 1827-1830 (2008).

5 Weaver, I. C., Cervoni, N., Champagne, F. A., D’Alessio, A. C., Sharma, S., Seckl, J. R. et al. Epigenetic programming by maternal behavior. Nat. Neurosci. 9, 847-854 (2004).

6 Franklin, T. B., Russig, H., Weiss, I. C., Gräff, J., Linder, N., Michalon, A. et al. Epigenetic transmission of the impact of early stress across generations. Biol. Psychiatry 68, 408-415 (2010). 